Final submitted version of paper subsequently published as: Heffernan, T., Morrison, D., Magne, P., Payne, S. and Cotton, D.R.E (2018) Internalising Internationalisation: Views of internationalisation of the curriculum among non-mobile home students.

Studies in Higher Education

\title{
Internalising Internationalisation: Views of internationalisation of the curriculum among non-mobile home students
}

Troy Heffernan\#, Dave Morrison, Polly Magne, Simon Payne, Debby

\section{Cotton*}

University of Plymouth, Plymouth, UK

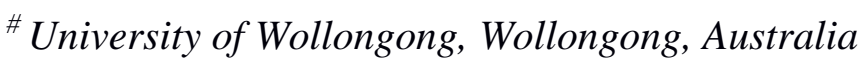

Provide full correspondence details here including e-mail for the corresponding author

* Corresponding author

Professor Debby Cotton,

3 Endsleigh Place,

Plymouth, UK

E-mail: dcotton@plymouth.ac.uk

Telephone: 01752587614 
Final submitted version of paper subsequently published as: Heffernan, T., Morrison, D., Magne, P., Payne, S. and Cotton, D.R.E (2018) Internalising Internationalisation: Views of internationalisation of the curriculum among non-mobile home students. Studies in Higher Education

\title{
Internalising Internationalisation: Views of internationalisation of the curriculum among non-mobile home students
}

\begin{abstract}
There is considerable literature on internationalising the curriculum in higher education, but relatively little of this focuses on developing international perspectives in home-based students. In particular, there has been limited direct engagement with student views and understandings, especially comparatively across a wide range of subjects. To address this gap, we surveyed 495 first-year students in nine subjects across four faculties at a UK university, asking about their views on internationalisation. We also examined an apparent bias in the literature towards Business subject case studies. We found some support for generic approaches to teaching internationalisation for home students, but a more widespread sense of the need for subject-based contextualisation. We also found that experiential learning models favoured in the Business-centric literature do not match the needs of students more widely.
\end{abstract}

Keywords: internationalisation, home students, internationalisation of the curriculum

\section{Introduction}

Internationalisation of the curriculum ( $\mathrm{IoC}$ ) is increasingly regarded as an essential aspect of higher education (HE), for both social and employability reasons. It appears as a core aim in the prospectuses and strategies of nearly all UK universities, for example. A wealth of literature on how to address IoC has emerged in the last few decades; most looking at the experience of international students, offshore campuses, and home students abroad. Yet the overwhelming majority of students, especially in the UK, are none of these. In 2016-17 only 19\% of students in UK universities were from outside the UK, and in 2015-16 only $1.6 \%$ of UK students travelled abroad for study or work placement (Higher Education Statistics Agency 2018; Go International 2018).

This discrepancy has sparked a growing interest in 'internationalisation at home' (IaH); developing intercultural/international awareness locally for home-based students. 
Final submitted version of paper subsequently published as: Heffernan, T., Morrison, D., Magne, P., Payne, S. and Cotton, D.R.E (2018) Internalising Internationalisation: Views of internationalisation of the curriculum among non-mobile home students. Studies in Higher Education

This pragmatic focus is laudable, but there are some important gaps in the current literature on $\mathrm{IaH}$, which this paper aims to help resolve. IaH research has focused chiefly on staff perceptions and experiences. There has been very little research on how home students themselves perceive internationalisation and, most importantly, how these views impact on the pedagogies necessary to develop an international approach in different subjects.

Additionally, there is a paucity of studies which look comparatively across multiple subjects. A considerable bias towards Business subjects as case studies has been noted in other parts of the internationalisation literature (Chan 2011). If a significant subject-bias also exists in the IaH literature, then pedagogic recommendations may not apply to students studying all subjects. Indeed, they may even hinder IaH learning.

To address these gaps, we surveyed the views on internationalisation of 495 first-year students at the start of their degrees, comparatively across nine subjects spanning four faculties at a major UK university. Further, we conducted a systematic search of the IaH literature to examine any bias. This paper focuses on answering three questions:

1: Across a broad range of subjects, what views and understandings of internationalisation do undergraduate home students enter HE with, and do these suggest any generic pedagogies for developing IaH?

2: Are there significant subject-level differences in the views and understandings of internationalisation that undergraduate home students enter $\mathrm{HE}$ with, and do these suggest any subject specific pedagogies for developing IaH? 
Final submitted version of paper subsequently published as: Heffernan, T., Morrison, D., Magne, P., Payne, S. and Cotton, D.R.E (2018) Internalising Internationalisation: Views of internationalisation of the curriculum among non-mobile home students. Studies in Higher Education

3: To what extent is there a bias in the IoC/IaH literature towards particular subjects, and do the views and understandings of undergraduate home students across a range of subjects justify this as representative?

We specifically sought to examine home student views at the start of their university career. Understanding their perspectives at this stage can help identify effective pedagogies to engage students early and set a strong course for further development. For example, do some students enter higher education already actively resistant to learning about internationalisation (and correlate ideas such as multiculturalism and global citizenship) for subject-specific reasons, or are most students tabula rasa that would suggest generic policies and pedagogies would be effective? Even otherwise effective generic pedagogies, such as those proposed in the current literature, may leave students confused or disengaged if we have not first considered their pre-conceptions and expectations.

\section{Background}

\section{Defining Internationalisation at Home}

The term 'Internationalisation at home' (IaH) was first coined in 1999, but remained mostly within a continental European context for some time, only recently becoming a prominent focus within the UK literature (Jones and Killick 2007). Definitions of internationalisation of the curriculum (IoC) in a broad sense show subtle, but critical, differences from IaH more specifically. Leask $(2009,209)$ defined IoC as 'the incorporation of an international and intercultural dimension into the preparation, delivery and outcomes of a program of study'. Knight (2008, ix) offered the most recent iteration of a definition that has evolved since 1994, '...the process of integrating an international, intercultural, and global dimension into higher education's major 
Final submitted version of paper subsequently published as: Heffernan, T., Morrison, D., Magne, P., Payne, S. and Cotton, D.R.E (2018) Internalising Internationalisation: Views of internationalisation of the curriculum among non-mobile home students. Studies in Higher Education

functions and delivery modes at both the institutional and national levels'. Definitions of $\mathrm{IaH}$, in a sense, both broaden and narrow the focus of IoC, e.g. 'the purposeful integration of international and intercultural dimensions into the formal and informal curriculum for all students, within domestic learning environments' (Beelen and Jones 2015b, 8). This definition, which we use here, does not isolate home students from international students, but adds them explicitly into the picture.

\section{Subject-Specific or Generic Pedagogies}

An enduring discussion in the IoC/IaH literature is whether generic or subjectspecific pedagogies are most effective. Working with teaching staff across a range of subjects, Leask $(2012,28)$ concluded that the vicissitudes of disciplinarity meant that IaH must primarily be handled case by case within the subjects. Clifford (2009) came to similar conclusions, and Watkins and Smith (2018) identified how IaH employability skills can be enhanced through creative course design. These conclusions are based on considerable differences in subject-level staff views about the relevance of IoC/IaH. While staff in some subjects, particularly Business, were consistently positive about IoC/IaH, staff in other subjects offered strong resistance, for example asserting that '...university policies on internationalization didn't apply to their program; in some [subjects], staff claimed that what was happening in other parts of the world was of no relevance to what they were teaching' (Leask 2013, 113; Childress 2010).

Conversely, some authors have focused on more generic approaches including centralised internationalisation courses, non-disciplinary optional programmes, or broad implementation of internationalisation for home students via graduate attributes (Jones and Killick 2013; Soria and Troisi 2013; Jones 2014). Yet others call for a balance between generic and subject-specific solutions (Killick 2015; Beelen and Jones 2015c). Leask (2013) argued that, in conjunction with subject-specific pedagogies, centralised 
Final submitted version of paper subsequently published as: Heffernan, T., Morrison, D., Magne, P., Payne, S. and Cotton, D.R.E (2018) Internalising Internationalisation: Views of internationalisation of the curriculum among non-mobile home students. Studies in Higher Education

institutional coordination and collaboration with Educational Developers was necessary to mitigate the resistance and division among staff at the subject level. Similarly, de Haan and Sherry (2012) identified the difficulties IaH, at subject level, can face without an accepting institutional environment.

What has not been significantly addressed in the literature, however, is how home-based students see internationalisation in relation to their subjects, and what this tells us about the most effective pedagogies.

\section{Experiential learning and Internationalisation at Home}

Over the past decade, there has been a clear focus in the IaH literature on 'experiential learning' (Wang et al. 2014). Broadly, this refers to students directly 'experiencing' something international or multicultural. Jones (2014) stresses developing new ways to encourage international experiences for home students in order to make them more globally employable. In its simplest form, this involves actively integrating existing international students with home students, such as by using group work engineered to include international and home students in each group (Brown 2013). 'Experience', however, often extends beyond the classroom, or even the campus, in an effort to account for differing levels of international students present in different institutions or subjects. Beelan and Jones $(2015 \mathrm{a}, 13)$ propose the value of '... working with local cultural, ethnic, or religious groups; using a tandem learning system or other means to engage domestic with international students; or exploiting diversity within the classroom.' Institutional advice frequently replicates this view that direct engagement with international experiences is crucial: 'an important part of internationalisation at home lies in creating a vibrant international community for learning and research' (Fielden and Leadership Foundation for Higher Education 2011, 39). 
Final submitted version of paper subsequently published as: Heffernan, T., Morrison, D., Magne, P., Payne, S. and Cotton, D.R.E (2018) Internalising Internationalisation: Views of internationalisation of the curriculum among non-mobile home students. Studies in Higher Education

Arguably, this experiential learning focus reflects an underlying bias in the literature towards subjects where such opportunities are more present and students are likely to engage with them, specifically Business subjects. Indeed, Chan (2011) noted a considerable bias towards Business subjects within the literature on the similar topic of transnational education. Such a focus would not be hard to understand; Business subjects accounted for $15.2 \%$ of the total incoming international students to the UK in 2014-15, significantly more than the next highest subject at 10\% (Engineering), and many times more than most others (HESA 2016). Of the $1.3 \%$ of UK students who studied abroad, $13.3 \%$ were from Business in in 2014-15; the next highest subject-area accounted for only 6.9\% (HESA 2016).

In principle, there is nothing troubling about focusing research where an issue is most relevant. However, if there is a Business bias in the IaH literature, it could suggest that the prevalence of experiential learning reflects conditions within Business courses and the views of Business students which may not reliably represent the situation of home students more broadly. For example, it is difficult, but not impossible, to see how experiential approaches such as engaging with local multicultural settings would be made contextually relevant to some subjects (e.g. Maths, Chemistry). Further, it is not obvious that students in other subjects would engage positively with these pedagogies; we have already seen that research on staff in a range of disciplines suggests the opposite.

\section{Method}

In the first stage of this study, we used the ERIC, EmeraldInsight, and British Education Index journal databases to identify relevant papers published between January 2010 and April 2016, using the keywords 'internationalization', 'global citizenship', and 'crosscultural competence'. These were the most ubiquitous terms encountered in our 
Final submitted version of paper subsequently published as: Heffernan, T., Morrison, D., Magne, P., Payne, S. and Cotton, D.R.E (2018) Internalising Internationalisation: Views of internationalisation of the curriculum among non-mobile home students. Studies in Higher Education

preliminary research. Results were filtered for those relating to 'higher education' using options available in the search parameters. This identified 399 papers on internationalisation of the curriculum. Searches of additional databases did not yield significant unique sources. The abstracts and methods were then systematically reviewed to identify papers presenting empirical studies of teaching or curriculum design for internationalisation and/or student experiences, but which were not exclusively focused on study abroad or international student support.

To evaluate student views of IaH, we surveyed 495 first-year students at the end of their first module (week four) in nine subjects (disciplines) across four faculties at a major UK university:

$\underline{\text { Subject }}$

- Primary Education (Education)

- English Literature (English Lit.)

- Social Work

- Chemistry

- Maths

- Biology

- Software Development (Computing)

- Marketing

- Tourism \& Hospitality (Tourism)
Faculty

Arts \& Humanities

Arts \& Humanities

Health \& Human Sciences

Science \& Engineering

Science \& Engineering

Science \& Engineering

Science \& Engineering

Business

Business

Our sampling was purposive, with the express aim of having at least one representative from each Faculty (Bryman 2015). To gain representative student views, we administered the surveys in class during the final session of each module. This mitigated problems with self-selection and low response rates often faced by online surveys (Duda and Nobile 2010). Table 1 shows the response rates and demographic data for each subject. Class sizes varied substantially, and our analysis of aggregate trends was weighted accordingly. 
Final submitted version of paper subsequently published as: Heffernan, T., Morrison, D., Magne, P., Payne, S. and Cotton, D.R.E (2018) Internalising Internationalisation: Views of internationalisation of the curriculum among non-mobile home students. Studies in Higher Education

The first-year structure at the institution was advantageous in that students are only enrolled in a single subject-specific 'immersive' module for the first four weeks, ensuring that any links between student views and teaching methods were not influenced by other modules. Further, all incoming students in a subject are enrolled together, meaning that our survey was issued to the full first-year cohort for each subject. Notably, $89.4 \%$ of first-year students at the institution were UK domiciled (i.e. home students).

The surveys consisted of mostly Likert-scale questions asking students to indicate their agreement with statements about internationalisation in the curriculum (Table 2). Of these 'viewpoint' questions, five were directly positive about internationalisation, two were directly negative, and two asked about external conditions (international students or study abroad). The two external questions were coded as negative also, because agreement suggested a view that internationalisation cannot be learned without the presence of international students or travelling abroad.

Students were also asked to report particular IoC related pedagogies that they did or did not experience. Module leads in each subject in our study confirmed that there was no explicit discussion of IoC/IaH, multiculturalism, or global aspects of the subject within the introductory 4-week modules. Students were inducted into their subjects, but not explicitly to internationalisation. Therefore, our questions to students regarding pedagogies they observed aimed to uncover implicit internationalisation.

\section{[Table 2]}

By design, we did not define or discuss the term 'internationalisation', or any correlates such as multiculturalism or global citizenship, in advance. Further, we presented the survey in as neutral a context as possible. Our objective was to gauge student reaction to internationalisation and related ideas however they chose to define 
Final submitted version of paper subsequently published as: Heffernan, T., Morrison, D., Magne, P., Payne, S. and Cotton, D.R.E (2018) Internalising Internationalisation: Views of internationalisation of the curriculum among non-mobile home students. Studies in Higher Education

these terms and whether or not they were already familiar with them. Our choice of analysis method reflects this decision. We did not assume or expect uniform understandings, especially across subjects. As such, we avoided parametric measures (e.g. ANOVA and regression), which assume uniformity within and across groups since this is rarely justifiable across different academic subjects (Jamieson 2004; Cohen et al. 2011). We chose a 6-option Likert scale, intentionally avoiding a neutral option to discourage non-reflective responses.

Negatively coded questions were reversed for analysis, such that in the discussion below 'disagreement' with these indicates that the student agreed with the negative statement (i.e. that they disagreed with a positive rephrasing of it).

To compare student views to reported teaching methods, we employed bivariate correlation using Kendall's Tau rank correlation test ( $\left.\tau_{\mathrm{b}}\right)$ using SPSS 22. As noted, these are non-parametiric measures which do not presume uniform understanding of the scale across different groups. Rank correlation relies on the relation of median scores in ordinal data, where mean scores cannot be presumed to be consistent or reliable, such as with Likert scales. Further, we have reported quartiles along with the median values since Likert data can be ambiguous from the median alone (the same median could indicate polarised student views or centralised ones) (Clegg 1997). We evaluated the effect sizes of the correlations according to Cohen's classification of r-values, meaning that correlations of $\left(\tau_{b}=.1-.299\right)$ were considered 'small' effects, $\left(\tau_{b}=.3-.499\right)$ 'medium', and $\left(\tau_{\mathrm{b}}>=.5\right)$ 'high' (Gray and Kinnear, 2012). Correlations below $\left(\tau_{\mathrm{b}}=.1\right)$, even if significant, were considered too weak to be meaningful.

To examine specific trends across questions and subjects, we conducted principal factor analysis (PFA) and two-way cluster analysis, using SPSS 22. PFA aided in isolating questions whose answer patterns cohered with other questions to form 
Final submitted version of paper subsequently published as: Heffernan, T., Morrison, D., Magne, P., Payne, S. and Cotton, D.R.E (2018) Internalising Internationalisation: Views of internationalisation of the curriculum among non-mobile home students. Studies in Higher Education

groups, and to identify any outliers. Two-way cluster analysis, conversely, allowed us to identify patterns of responses by subject which cohered as groups across the questions within each factor set.

The PFA revealed two sets within the viewpoint questions, a 'core' group including all five positive questions and 'Learning about different cultures is not relevant to my subject', and an 'external' group consisting of the two external questions. Internal consistency of each factor set was tested via Cronbach's alpha, presuming the convention of .7 or greater as a sufficient score (Moore and McCabe 2000). The 'core' set was confirmed $(\alpha=.765)$, however, Cronbach's alpha was quite low for the 'external' factor set $(\alpha=.444)$, suggesting that although these two questions cohere as a factor, there remains significant variance within the answers themselves (Grau, 1998). Factor analysis of the pedagogy questions showed only one group, confirmed also by a high Cronbach's score $(\alpha=.845)$, indicating high internal consistency between questions.

Students' final comments were coded thematically as either positive or negative towards internationalisation (Bryman 2015). Comments disputing the value of the survey were coded as negative, in the sense that they generally suggested that internationalisation was irrelevant, usually at the subject level.

\section{Results}

\section{Review of the IaH Literature}

Our review of 399 abstracts revealed that the largest proportion (168) dealt with international students, home students abroad, or transnational education. A further 115 were only indirectly related to students (e.g. broad policy discussions). Of the 137 empirical studies which did focus on internationalisation at home in some way, $21 \mathrm{did}$ 
Final submitted version of paper subsequently published as: Heffernan, T., Morrison, D., Magne, P., Payne, S. and Cotton, D.R.E (2018) Internalising Internationalisation: Views of internationalisation of the curriculum among non-mobile home students. Studies in Higher Education

not specify the subject(s) involved in the research. The subject or subjects covered in the remaining 116 are summarised in Table 3.

\section{[Table 3]}

A bias towards Business subjects is clear; nearly half of the IaH research relied exclusively on Business subjects as data sources. The next largest single subject area accounted for barely more than 1/5 of what Business subjects did. Social Sciences, Arts, and Humanities combined (excluding Education) accounted for only 11\%, with no single subject here accounting for more than $1 \%$ of the total. Comparative studies across multiple subjects were also infrequent compared to business-centric research, however, there were more of these that any other single subject and more have appeared in the latter 5 years than the former 11, which may indicate a trend towards taking a wider view.

\section{Student Views across Subjects}

Table 4 and figure 1 summarise the aggregate student views on internationalisation across all nine subjects together:

\section{[Table 4]}

\section{[Figure 1]}

Crucially, the majority of students were positive about internationalisation - regardless of subject or how they personally defined the terms. In this light, all discussion below of 'negative' student views must be seen as relative to this overall positive outcome. Five questions though, did have lower quartiles in the 'disagree' range, meaning that at least $25 \%$ of students across all subjects responded negatively to these questions. 
Final submitted version of paper subsequently published as: Heffernan, T., Morrison, D., Magne, P., Payne, S. and Cotton, D.R.E (2018) Internalising Internationalisation: Views of internationalisation of the curriculum among non-mobile home students. Studies in Higher Education

We also wanted to see if positive or negative views correlated with any particular IoC/IaH teaching practices that students reported, recalling that these would be implicit only during the modules we reviewed. Correlations here at the aggregate level could indicate universally effective IaH pedagogies. Several clear patterns emerged from our bivariate tests, though most effect sizes were small. Use of 'examples from different cultures or places' $\left(\tau_{\mathrm{b}}=.103-.442, \mathrm{p}<.05\right)$ and discussion of 'how cultural awareness relates to the subject' $\left(\tau_{\mathrm{b}}=.107-.509, \mathrm{p}<.05\right)$ correlated relatively well with positive views, even having a few 'medium' and one 'high' effect on Cohen's scale, suggesting that these may indeed be universally pivotal factors in developing students' international outlooks. Further, these two pedagogies nearly mirrored each other score for score, suggesting that they go well together (or are variations on a single pedagogic approach). The statements, 'I learned that there is an important international context to my subject'; 'I was challenged to think globally about my subject'; and 'We were able to relate our assignments to different cultures if we wanted to' also correlated positively with several student views, though marginally so. These three methods also mirrored each other view-for-view, suggesting a similar pedagogic synergy. The small effect sizes suggest that most of the variance in student views were not a product of these pedagogies though, which is to be expected as no explicit IoC/IaH teaching took place.

\section{Students' Views within the Subjects}

In this section, we look at the differences and similarities that emerged at the subjectlevel, and how these compare to the aggregate findings. Figure 2 provides a visualisation of student views for each of the 'core' set of five positive viewpoint questions plus 'learning about different cultures is not relevant to my subject' by 
Final submitted version of paper subsequently published as: Heffernan, T., Morrison, D., Magne, P., Payne, S. and Cotton, D.R.E (2018) Internalising Internationalisation: Views of internationalisation of the curriculum among non-mobile home students. Studies in Higher Education

subject.

[Figure 2]

Several patterns in the student views of internationalisation were apparent at this level, either in individual subjects or groups of subjects. The uniform positivity seen in the aggregate responses is nearly eliminated, an occurrence known as Simpson's paradox. This refers to an apparently clear aggregate outcome actually being misleading or obscuring critical variations once additional 'lurking' factors are accounted for (Moore and McCabe 2001). In our case the lurking factor is the subject differences, which brought to light significant disparity not visible in the wider view.

This is best displayed by the cluster analysis of the 'core' questions. The analysis showed two clear groups, separated by degree of positivity towards internationalisation (figure 3).

[Figure 3]

There was a striking near-polar distinction between the subject groups of Marketing, Tourism, and Social Work (MTSW) showing overwhelmingly positive views, and Chemistry, Computing and Maths (CCM) being strongly negative. However, as with the overall outcomes, negative is a comparative term here only; the median student views in the 'negative' cluster, dominated by the CCM group, were still marginally positive for each question.

What distinguished the groups, rather, was a substantial subset of students in the CCM group who responded very negatively. This was particularly true of whether students thought learning about other cultures was relevant or that global citizenship should be an aim of higher education. Conversely, the student views in the MTSW group were very positive, with even the lower quartile being positive for several 
Final submitted version of paper subsequently published as: Heffernan, T., Morrison, D., Magne, P., Payne, S. and Cotton, D.R.E (2018) Internalising Internationalisation: Views of internationalisation of the curriculum among non-mobile home students. Studies in Higher Education

questions.

To a degree, the subject clusters above reinforce the traditional 'two-cultures' view of Science and Arts/Social Science subjects as distinct (Snow 1961). However, responses in the remaining subjects, Education, English Literature, and Biology challenged such a binary approach. Student views in these were more balanced/centralised than the other clusters, but what most disrupts any 'two-cultures' approach to IaH pedagogies was that English Literature aligned more closely with the CCM subjects than Biology, which leaned more towards the MTSW group.

The two external questions formed their own cohesive set in the factor analysis. They tell a different story of student views on internationalisation, one which returns us to the issue of the Business focus in the IaH literature and its relation to experiential pedagogies (figure 4). A clear majority of students in both Marketing and Tourism felt that international students and/or study abroad were necessary to engage with internationalisation. Conversely, across all other subjects at least $60 \%$ of students felt that neither was needed. In Social Work, English Literature and Chemistry this was over $80 \%$.

[Figure 4]

Comparison between student views and reported pedagogies told us little about what works for IaH at the subject level; there were no clear patterns. The correlations seen at the aggregate level did not appear for any subject except Biology. However, cluster analysis of the degree to which students reported each pedagogic method did show some subject-level patterns (Figure 5)

[Figure 5]

Moreover, Marketing students reported much lower levels of IaH teaching (further 
Final submitted version of paper subsequently published as: Heffernan, T., Morrison, D., Magne, P., Payne, S. and Cotton, D.R.E (2018) Internalising Internationalisation: Views of internationalisation of the curriculum among non-mobile home students. Studies in Higher Education

confirmed by the module lead), nearly the same as English Literature, though student views in these subjects differed substantially. Biology students reported less teaching about internationalisation in their module than English Literature, but were nevertheless more positive about it.

This is where we must turn to qualitative analysis of students' final comments to cast light on why some students were opposed to internationalisation. There were clear trends in the response rates and degree of positivity/negativity of the comments by subject (Table 5).

\section{[Table 5]}

The patterns here closely match those of student viewpoint responses by subject. The content of the responses tells us much more. Uniformly across the CCM group, and Biology, the nature of student resistance was the presumed universality of the subject content.

I have no idea what internationalness or cultural awareness has to do with a computing/programming module

Chemistry is an international subject and is interpreted by every scientist the same no matter what culture they come from

I study Maths, it is the same everywhere so there are no international or cultural aspects

It's Science, it should all be the same regardless of culture and so it's international without having to place emphasis on the 'internationalness' [Biology]

There were also a small number of positive comments about internationalisation left by students in these subjects: 
Final submitted version of paper subsequently published as: Heffernan, T., Morrison, D., Magne, P., Payne, S. and Cotton, D.R.E (2018) Internalising Internationalisation: Views of internationalisation of the curriculum among non-mobile home students. Studies in Higher Education

In Chemistry the international concept is mainly looking at the background of theories and how the authors' culture was influenced by this

Although Maths is a calculation subject, it is interesting to learn about the historical and cultural/political influence of what we are taught

English Literature students also expressed significant issues with internationalisation in the final comments. In fact, they did so more and more strongly than any other subject, although the quantitative responses in English Literature were more positive than CCM (but not Biology). English Literature students, however, gave quite different reasons for their negativity. They felt that the subject explicitly lacked an international scope:

I don't think this is massively relevant to English as a course. We don't do world literature at this university

Pick a subject that this is relevant to! Like languages or geography

Why is this survey relevant to English degree? Found it a waste of my time and too politically correct - political correctness gone mad.

Indeed, even a seemingly overt indication of the module's international scope did not resonate with all students, 'although our module was based on the Greek poem The Odyssey, there wasn't much need to consider global perspectives.'

These student views mirror those of some staff that Leask (2012) and Childress (2010) found in similar subjects. But the fact that our study engaged with students at the very start of their degree indicates that students arrive in $\mathrm{HE}$ with these views, rather than developing them throughout enculturation into their subjects. 
Final submitted version of paper subsequently published as: Heffernan, T., Morrison, D., Magne, P., Payne, S. and Cotton, D.R.E (2018) Internalising Internationalisation: Views of internationalisation of the curriculum among non-mobile home students. Studies in Higher Education

\section{Discussion}

Our study set out to look at three interrelated questions about how students view internationalisation in different subjects, and what this may mean for designing effective pedagogies for internationalisation at home. Looking first at the reliability of generalising IaH pedagogies from the extant literature, Business subjects accounted for almost $50 \%$ of the IaH teaching literature. However, it was clear from the student data that there were significant, pedagogically relevant, differences in student views across subjects. Business students did cohere somewhat with other Social Science students (Social Work and Education) in having very positive views on the value of internationalisation overall, but this did not extend to Science or Humanities.

More critically, Business students held opposing views to all other subjects in their belief that international students and/or study abroad were necessary for learning about internationalisation. The literature, based on Business examples, strongly favours experiential learning models aimed at students directly engaging with international or multicultural situations, often on a volunteer or extra-curricular basis. Our study challenges the broad efficacy of this approach. We do not dispute the value of experiential learning itself. Indeed, there is no shortage of compelling evidence of its effectiveness (Killick 2012; Jones and deWit 2012). Rather, our data casts doubt upon this approach as a sole or even major mode for IaH.

There is a wide subject-based disparity in the presence of international students and uptake of study abroad, although some international students are present in all subjects, and all subjects allow for some form of study abroad at the institution. The Faculty of Business represented $49 \%$ of the total first-year international students at the institution; aside from Engineering (20\%), no other subject accounted for more than 
Final submitted version of paper subsequently published as: Heffernan, T., Morrison, D., Magne, P., Payne, S. and Cotton, D.R.E (2018) Internalising Internationalisation: Views of internationalisation of the curriculum among non-mobile home students. Studies in Higher Education

$10 \%$. This condition is indeed well-recognised and addressed in the IaH literature, via localised campus and community activities.

Within the Social Sciences at least, our findings indicate that experiential pedagogies such as these should be effective from the start. Business subjects, however, seem poor exemplars for effective pedagogies across Arts, Humanities, and Science subjects. Students in Maths, Chemistry, Computing, and English Literature were not inherently amenable to learning about internationalisation. Among the CCM group, students' qualitative and quantitative responses aligned - a substantial minority in each subject rejected the relevance of internationalisation to their subject. English Literature students were quantitatively more in favour of internationalisation, while simultaneously having more and stronger comments against it than any other subject. Further, the use of multicultural examples in teaching, though correlated broadly with positive student views, was significantly less effective (or present) in these subjects. Together, these make even localised multicultural approaches such as volunteering and campus events likely to meet early resistance. Indeed, to engage students at all, important ground-work would be needed in subjects like Maths or English Literature to develop students' awareness of how internationalisation principles are relevant to their subject. Introducing experiential pedagogies without this foundation could lead to disengagement, poor satisfaction, and retention issues, in addition to simply failing to achieve the aims of developing global and multicultural thinking about their subject. The first hurdle must be convincing students that internationalisation is something they even should be made aware of, which requires a considerable broadening of the assumptions present in the Business-based models.

If not experiential learning, then what? Did our data offer any solutions or new insight into effective pedagogies that would help develop IaH in the more resistant 
Final submitted version of paper subsequently published as: Heffernan, T., Morrison, D., Magne, P., Payne, S. and Cotton, D.R.E (2018) Internalising Internationalisation: Views of internationalisation of the curriculum among non-mobile home students. Studies in Higher Education

subjects? Looking first at generic pedagogies, we saw that resistance to IaH was not a majority view even in Science subjects. To a degree this challenges the idea that internationalisation cannot be embedded generically in HE - through approaches such as a graduate attributes framework (Jones and Killick 2013). Indeed, such an approach appears to counter some of the more intense subject-specific negativity apparent in staff-focused studies (e.g. Childress 2010; Leask 2012). Given the overall positive student views, well-designed IaH initiatives should have some traction in any subject if introduced early and in a considered manner. Encountered resistance thus may stem from inappropriate design rather than broad student disengagement.

In terms of effective pedagogies, contextualised instruction about internationalisation correlated significantly with positive views, particularly where examples from other cultures were used or the relevance of cultural awareness to the subject was made clear. Despite the fact that these approaches were subject-based, the generic pedagogic principle which emerges is that providing explicit instruction for students about internationalisation is advantageous. Clearly signposting to students in any subject that they are learning about internationalisation and why this is important is crucial in enhancing understanding and engagement.

The relatively small number of negative student views which emerged broadly mirrored the reactions of staff in other studies - although these seem likely to be preexisting student views, rather than being imparted by staff. In this, our findings support the literature in favour of subject-specific pedagogies (Leask 2012; Beelen and Jones 2015c). Within the Social Sciences, the current trend for experiential learning seems likely to succeed well from the start. In other subjects preparation is needed first to engage the views of students who don't initially see the relevance of internationalisation. As a majority of students even in these subjects held positive 
Final submitted version of paper subsequently published as: Heffernan, T., Morrison, D., Magne, P., Payne, S. and Cotton, D.R.E (2018) Internalising Internationalisation: Views of internationalisation of the curriculum among non-mobile home students. Studies in Higher Education

views, one approach may be to leverage this via group-based projects with a subjectspecific international element. This would allow students to learn about the relevance of the topic from their peers. Another approach may be to introduce an international feature of the subject within the assessment. For example, the Maths module lead suggested asking students to address how approaches to cryptography (the module focus) may be influenced by different social and legal conditions globally. This would address the international aspects of the subject without directly challenging some students' perceptions that the content itself is universal. It could therefore be an effective bridge towards students seeing the relevance of experiential pedagogies.

English Literature students had very different reasons for resisting IoC ideas. They felt that the subject intrinsically focused on the national level. Pedagogies here might include a series of readings from English writers who came from other cultures, or wrote about other cultures, or critiques of English Literature from other cultures. The module we reviewed focused on Homer's Odyssey, not itself English literature at all. The module leader suggested a simple approach of directly discussing how this and other non-English sources formed the foundations of English literature. This aligns well with our finding that explicit reference to international context may be the most important bridging element after subject relevance.

Further research evaluating the impact of introducing new pedagogic approaches, such as those described above, would be beneficial, as would an expanded study which included Technology, Medicine, and Fine Arts subjects. Our study has added much-needed student facing data to both validate and challenge current trends in the internationalisation at home literature. Pedagogies designed to contextualise international aspects of each subject appear to be necessary to engage with student views effectively. Further, our comparative analysis has highlighted a strong disconnect 
Final submitted version of paper subsequently published as: Heffernan, T., Morrison, D., Magne, P., Payne, S. and Cotton, D.R.E (2018) Internalising Internationalisation: Views of internationalisation of the curriculum among non-mobile home students. Studies in Higher Education

between the evidence used to develop IaH pedagogies and the actual views of students in some subjects. We have provided an important addition to the literature, suggesting ways in which universities can more effectively develop a sense of positive international awareness, seen as so vital for students as future leaders. 
Final submitted version of paper subsequently published as: Heffernan, T., Morrison, D., Magne, P., Payne, S. and Cotton, D.R.E (2018) Internalising Internationalisation: Views of internationalisation of the curriculum among non-mobile home students.

Studies in Higher Education

References

Beelen, Jos, and Elspeth Jones. 2015a. "Europe Calling: A New Definition for Internationalization at Home." International Higher Education, no. 83: 12-13. . 2015b. "Looking Back at 15 Years of Internationalisation at Home." Forum. . 2015c. "Redefining Internationalization at Home." In The European Higher Education Area: Between Critical Reflection and Future Policies: Part 1, edited by Adrian Curaj, Liviu Matei, Remus Pricopie, Jamil Salmi, and Peter Scott.

Brown, Sally. 2013. "Internationalising Assessment, Learning and Teaching." AISHE-J: The All Ireland Journal of Teaching and Learning in Higher Education 5 (2).

Chan, B. 2011. "Postgraduate Transnational Education in Nonbusiness Subjects: Can It Fit Conceptualizations of Curriculum Internationalization?" Journal of Studies in International Education 15 (3): 279-98.

Childress, Lisa K. 2010. The Twenty-First Century University: Developing Faculty Engagement in Internationalization. Vol. 32. Peter Lang.

Clifford, Valerie Anne. 2009. "Engaging the Disciplines in Internationalising the Curriculum." International Journal for Academic Development 14 (2): 133-43.

de Haan, Donna, and Emma Sherry. 2012. "Internationalisation of the Sport Management Curriculum: Academic and Student Reflections." Journal of Studies in International Education, 16 (1): 24-39.

Dolnicar, S., and B. Grun. 2008. "Challenging 'Factor-Cluster Segmentation.'” Journal of Travel Research 47 (1): 63-71.

Fielden, John, and Leadership Foundation for Higher Education (Great Britain). 2011. Getting to Grips with Internationalisation: Resources for UK Higher Education Institutions. London: Leadership Foundation for Higher Education.

Go International. 2018. Latest mobility facts and figures http://go.international.ac.uk/content/how-many-students-are-going-abroad-uk.

Grau, Eric. 1998 Using Factor Analysis and Cronbach's Alpha to Ascertain Relationships Between Questions of a Dietary Behavior Questionnaire.

Higher Education Statistics Agency. 2018. Higher Education Student Statistics: UK, 2016/17 - Summary. https://www.hesa.ac.uk/news/11-01-2018/sfr247-highereducation-student-statistics.

Jamieson, Susan. 2004. "Likert Scales: How to (Ab)use Them." Medical Education 38 (12): 1217-18.

Jones, Elspeth. 2014. "Graduate Employability and Internationalization of the Curriculum at Home." International Higher Education, no. 78: 6-8.

Jones, Elspeth, and David Killick. 2013. "Graduate Attributes and the Internationalized Curriculum: Embedding a Global Outlook in Disciplinary Learning Outcomes." Journal of Studies in International Education 17 (2): 165-182.

Jones, Elspeth, and Hans de Wit. 2012. "Globalization of Internationalization: Thematic and Regional Reflections on a Traditional Concept." AUDEM: The International Journal of Higher Education and Democracy 3 (1): 35-54.

Killick, David. 2012. "Seeing Ourselves-in-the-World: Developing Global Citizenship through International Mobility and Campus Community." Journal of Studies in International Education 16 (4): 372-89.

Killick, David. 2015. "Internationalisation and the Academic Developer." Educational Developments 16 (3): 1-6.

Knight, Jane. 2008. Higher education in turmoil: the changing world of internationalisation. Global perspectives on higher education 13. Rotterdam: Sense Publishing. 
Final submitted version of paper subsequently published as: Heffernan, T., Morrison, D., Magne, P., Payne, S. and Cotton, D.R.E (2018) Internalising Internationalisation: Views of internationalisation of the curriculum among non-mobile home students. Studies in Higher Education

Leask, Betty. 2009. "Using Formal and Informal Curricula to Improve Interactions Between Home and International Students." Journal of Studies in International Education 13 (2): 205-21.

Leask, Betty. 2012. "Fellowship Report: Internationalisation of the Curriculum in Action." Australian Government: Office of Teaching and Learning.

- 2013. "Internationalizing the Curriculum in the disciplines-Imagining New Possibilities." Journal of Studies in International Education 17 (2): 103-118.

Moore, D.S., and G.P. McCabe. 2000. Manual for Introduction to the Practice of Statistics. W. H. Freeman.

Snow, C.P. 1961. The Two Cultures and the Scientific Revolution New York: Cambridge University Press.

Soria, Krista M., and Jordan Troisi. 2013. "Internationalization at Home Alternatives to Study Abroad: Implications for Students' Development of Global, International, and Intercultural Competencies." Journal of Studies in International Education,

Wang, Dan, Taiwen Feng, Susan Freeman, Di Fan, and Cherrie Jiuhua Zhu. 2014. "Unpacking the 'skill - Cross-Cultural Competence' Mechanisms: Empirical Evidence from Chinese Expatriate Managers.” International Business Review 23 (3): $530-41$.

Watkins, Heather, and Roy Smith. 2018. "Thinking Globally, Working Locally: Employability and Internationalization at Home." Journal of Studies in International Education. First Published January 10, 2018. 
Final submitted version of paper subsequently published as: Heffernan, T., Morrison, D., Magne, P., Payne, S. and Cotton, D.R.E (2018) Internalising Internationalisation: Views of internationalisation of the curriculum among non-mobile home students. Studies in Higher Education

\begin{tabular}{|c|c|c|c|c|c|}
\hline & $\begin{array}{c}\text { Total } \\
\text { Responses }\end{array}$ & Female & Male & $\begin{array}{c}\text { Age } \\
\text { (avg.) }\end{array}$ & $\begin{array}{c}\text { Response } \\
\text { Rate }\end{array}$ \\
\hline Marketing & 45 & $45 \%$ & $55 \%$ & 19.1 & $73.8 \%$ \\
\hline Tourism & 35 & $77 \%$ & $23 \%$ & 19 & $71.4 \%$ \\
\hline Social Work & 24 & $87 \%$ & $13 \%$ & 27.9 & $42.1 \%$ \\
\hline Education & 124 & $73 \%$ & $27 \%$ & 19.9 & $79.5 \%$ \\
\hline English & 70 & $75 \%$ & $23 \%$ & 19.5 & $75.3 \%$ \\
\hline Biology & 77 & $59 \%$ & $41 \%$ & 21.1 & $45.6 \%$ \\
\hline Chemistry & 38 & $39 \%$ & $61 \%$ & 19.6 & $64.4 \%$ \\
\hline Computing & 30 & $0 \%$ & $100 \%$ & 19.8 & $76.9 \%$ \\
\hline \multirow[t]{2}{*}{ Maths } & 51 & $29 \%$ & $71 \%$ & 19.9 & $67.1 \%$ \\
\hline & Total & $57 \%$ & $41 \%$ & & $65 \%$ \\
\hline
\end{tabular}

Table 1. Demographics 
Final submitted version of paper subsequently published as: Heffernan, T., Morrison, D., Magne, P., Payne, S. and Cotton, D.R.E (2018) Internalising Internationalisation: Views of internationalisation of the curriculum among non-mobile home students.

Studies in Higher Education

'Viewpoint' Questions

\section{Positive}

- I expect a university level module in my subject to have a global/international scope. [Expected global scope]

- It is important to know about how other nations or cultures approach my subject. [Other cultural approaches]

- Global citizenship is one thing that higher education is for. [HE for global citizens]

- Students should have to be more culturally sensitive and aware. [Should be more aware]

- To get a good job in my subject you need to look at it globally. [Get a good job]

\section{Negative}

- In my subject, learning about internationalisation takes time away from learning more important skills. [IaH Distracting]

- Learning about different cultures is not relevant to my subject. [IaH Irrelevant]

\section{External}

- You need to have international students in your class to have a multicultural experience. [Need intl. students]

- You need to travel or study abroad to learn about international parts of my subject. [Need study abroad]

\section{Teaching Methods (student self-reported)}

- In this module, examples from different cultures or places were used to explain some points.

- In this module we discussed how cultural awareness relates to the subject.

- During this module I was introduced to points of view different from the ones I grew up with.

- During this module, we discussed upcoming opportunities for international or multicultural learning.

- In this module we learned that there is an important international context to my subject.

- In this module we could choose different assignments to do, or different ways to hand in the same assignments.

- In this module I was challenged to think globally about my subject.

- In this module we were able to relate our assignments to different cultures if we wanted to.

Table 2: Survey Questions

\begin{tabular}{|l|c|c|}
\hline \multicolumn{1}{|c|}{ Subject } & Total Papers & Percent \\
\hline Business & 55 & $47.4 \%$ \\
\hline Multiple Subjects Compared & 17 & $14.7 \%$ \\
\hline Education & 11 & $9.4 \%$ \\
\hline Science & 8 & $6.9 \%$ \\
\hline Engineering & 6 & $5.2 \%$ \\
\hline Health/Medicine & 6 & $5.2 \%$ \\
\hline Other Single Subjects (Social Science, Arts and Humanities) & 13 & $11.2 \%$ \\
\hline
\end{tabular}

Table 3: Focus of the Literature 
Final submitted version of paper subsequently published as: Heffernan, T., Morrison, D., Magne, P., Payne, S. and Cotton, D.R.E (2018) Internalising Internationalisation: Views of internationalisation of the curriculum among non-mobile home students.

Studies in Higher Education

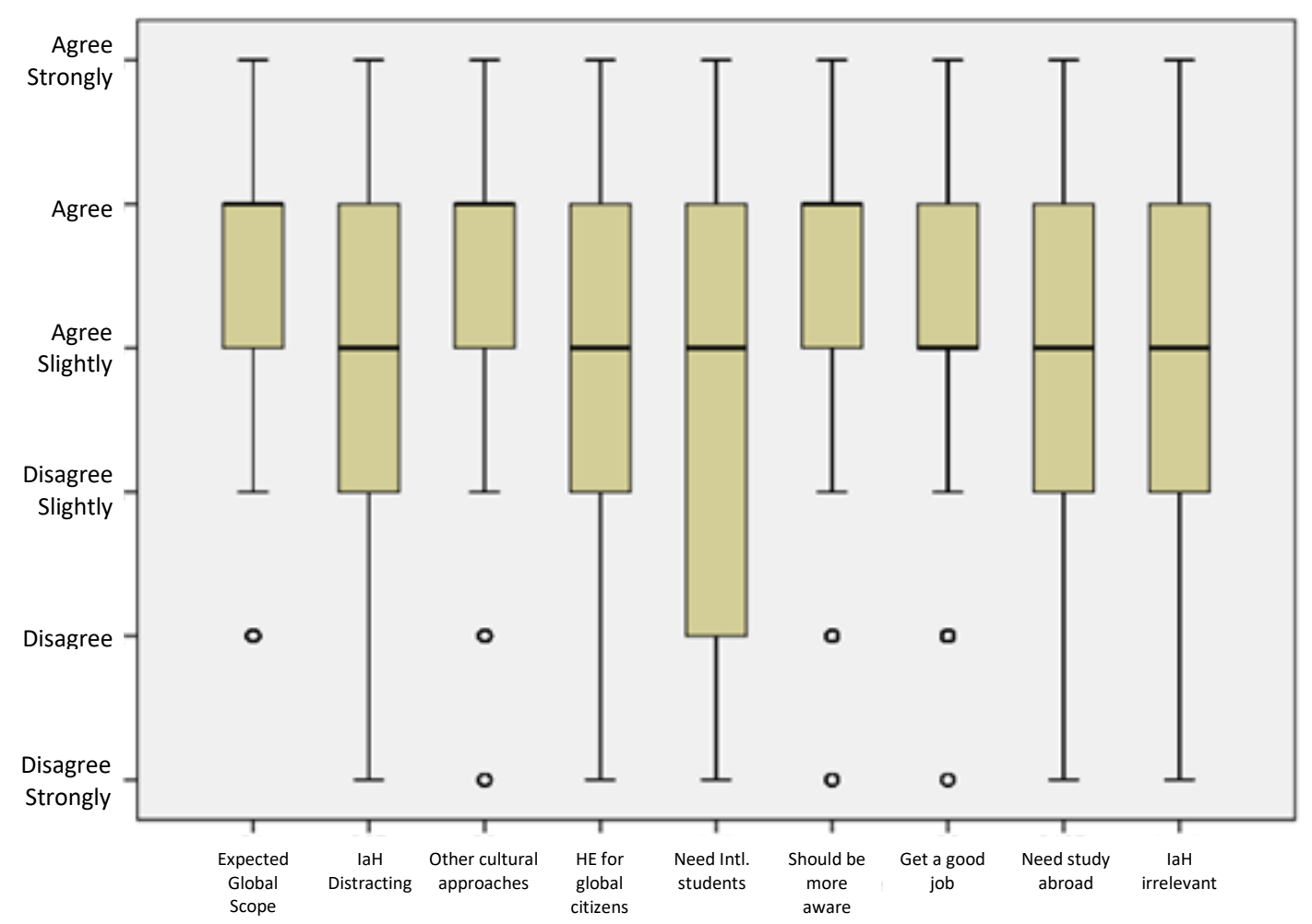

Figure 1: Aggregate Student Views

\begin{tabular}{|c|c|c|c|c|c|c|c|c|c|c|}
\hline & & $\begin{array}{l}\text { Expected } \\
\text { global } \\
\text { scope }\end{array}$ & $\begin{array}{c}\text { laH } \\
\text { distracting }\end{array}$ & $\begin{array}{c}\text { Other } \\
\text { cultural } \\
\text { approaches }\end{array}$ & $\begin{array}{l}\text { HE for } \\
\text { global } \\
\text { citizens }\end{array}$ & $\begin{array}{l}\text { Need } \\
\text { Intl. } \\
\text { students }\end{array}$ & $\begin{array}{c}\text { Should } \\
\text { be more } \\
\text { aware }\end{array}$ & $\begin{array}{l}\text { Get a } \\
\text { good } \\
\text { job }\end{array}$ & $\begin{array}{l}\text { Need } \\
\text { study } \\
\text { abroad }\end{array}$ & $\begin{array}{l}\text { laH } \\
\text { irrelevant }\end{array}$ \\
\hline \multirow{3}{*}{ Quartile } & 25 & 4.0 & 3.0 & 4.0 & 3.0 & 2.0 & 4.0 & 4.0 & 3.0 & 3.0 \\
\hline & Median & 5.0 & 4.0 & 5.0 & 4.0 & 4.0 & 5.0 & 4.0 & 4.0 & 4.5 \\
\hline & 75 & 5.0 & 5.0 & 5.0 & 5.0 & 5.0 & 5.0 & 5.0 & 5.0 & 5.0 \\
\hline \multicolumn{2}{|c|}{ Std. Deviation } & 1.1 & 1.2 & 1.2 & 1.1 & 1.5 & 1.1 & 1.2 & 1.3 & 1.6 \\
\hline
\end{tabular}

Table 4: Aggregate Student Views 
Final submitted version of paper subsequently published as: Heffernan, T., Morrison, D., Magne, P., Payne, S. and Cotton, D.R.E (2018) Internalising Internationalisation: Views of internationalisation of the curriculum among non-mobile home students.

Studies in Higher Education

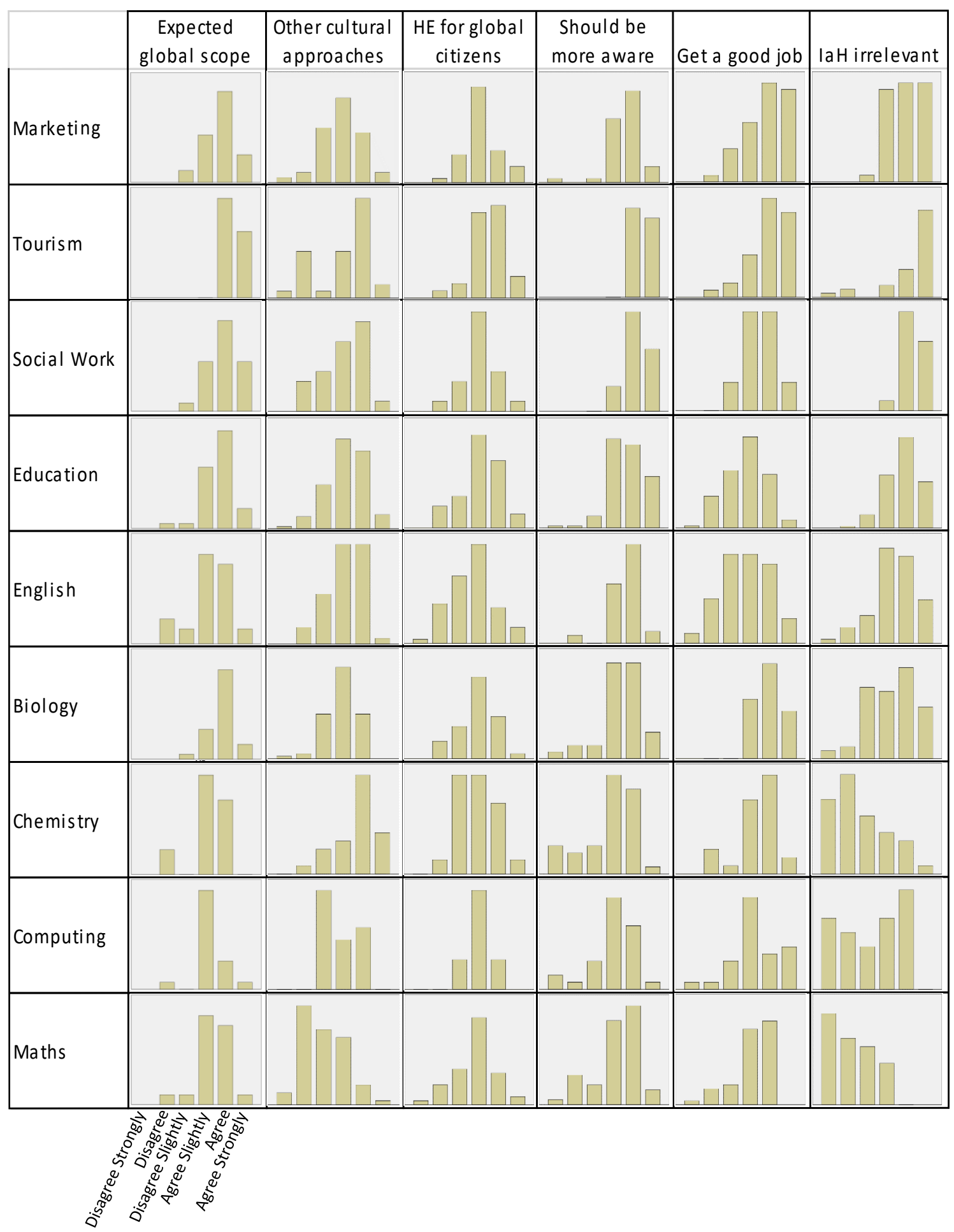

Figure 2: Subject-level Student Views - 'core' question set 
Final submitted version of paper subsequently published as: Heffernan, T., Morrison, D., Magne, P., Payne, S. and Cotton, D.R.E (2018) Internalising Internationalisation: Views of internationalisation of the curriculum among non-mobile home students.

Studies in Higher Education

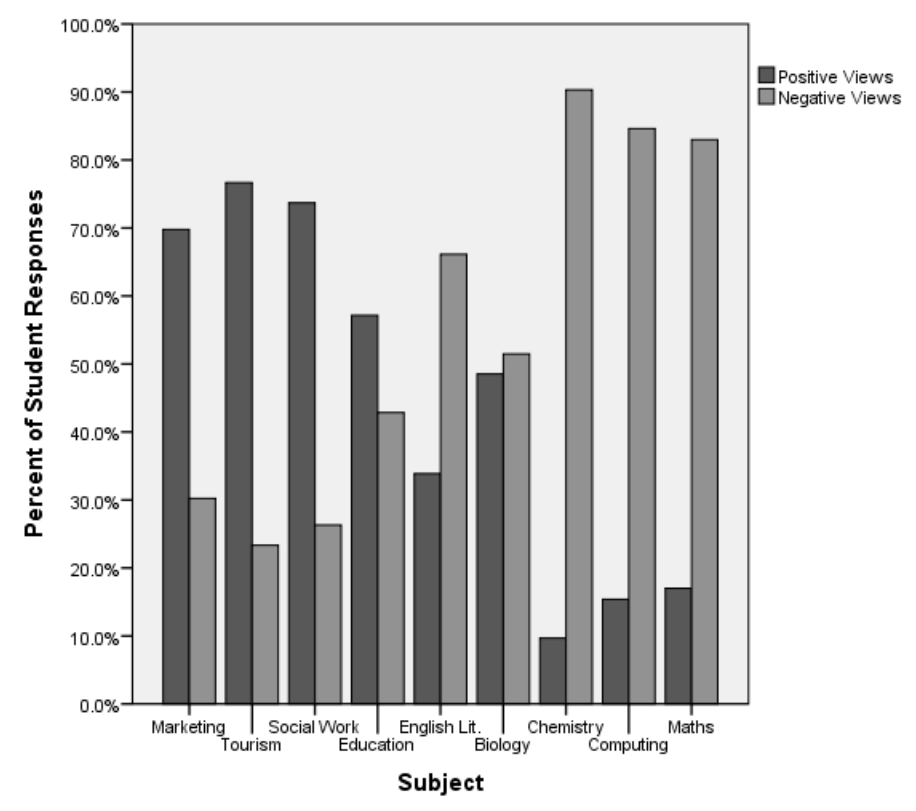

Figure 3: Core Viewpoint Subject Clusters

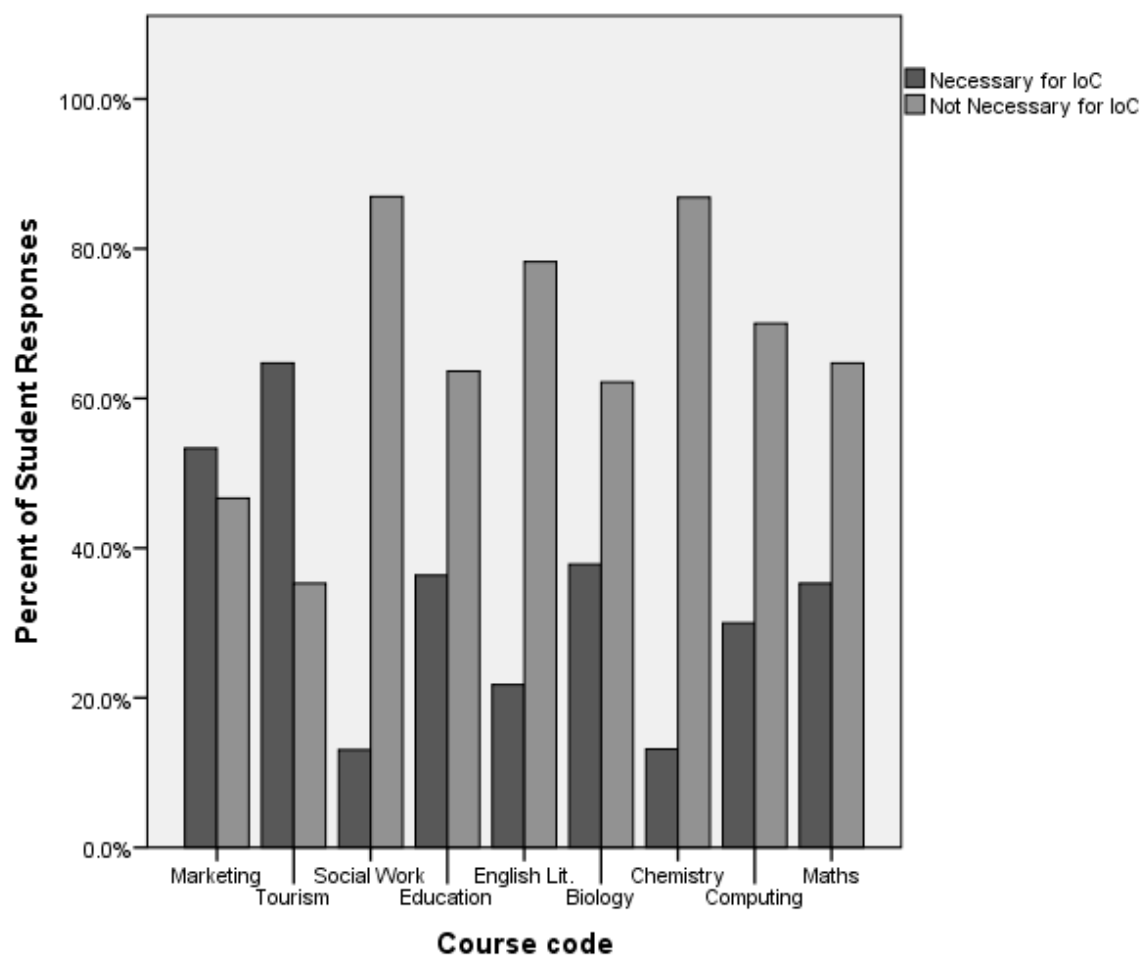

Figure 4: External Viewpoint Subject Clusters 
Final submitted version of paper subsequently published as: Heffernan, T., Morrison, D., Magne, P., Payne, S. and Cotton, D.R.E (2018) Internalising Internationalisation: Views of internationalisation of the curriculum among non-mobile home students. Studies in Higher Education

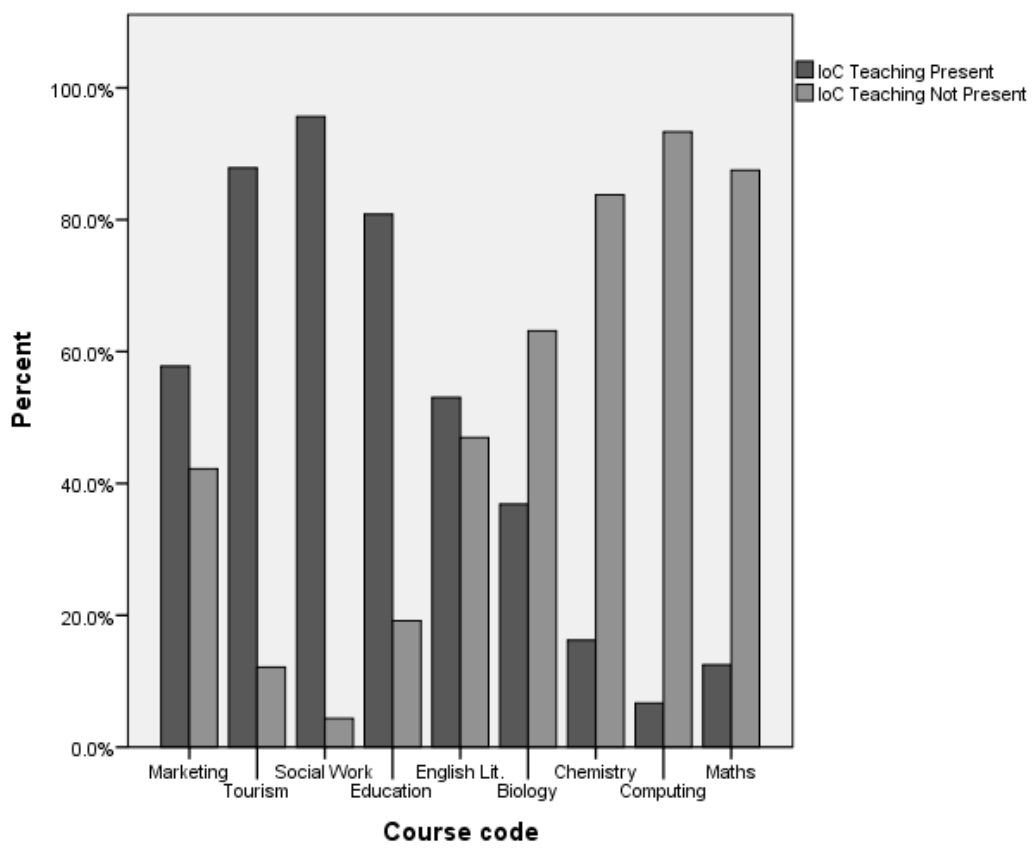

Figure 5: Teaching Method Subject Clusters

\begin{tabular}{|lcccc}
\multicolumn{1}{c}{ Subject } & Total Students & Response Rate & Negative & Positive \\
\hline Marketing & 45 & $6 \%$ & 0 & 3 \\
\hline Tourism & 35 & $3 \%$ & 0 & 1 \\
\hline Social Work & 24 & $12 \%$ & 0 & 3 \\
\hline Education & 124 & $5 \%$ & 1 & 4 \\
\hline English Lit. & 71 & $17 \%$ & 11 & 1 \\
Biology & 77 & $4 \%$ & 3 & 0 \\
Chemistry & 38 & $26 \%$ & 9 & 1 \\
\hline Computing & 30 & $20 \%$ & 5 & 1 \\
\hline Maths & 51 & $18 \%$ & 8 & 1
\end{tabular}

Table 5: Final Comment Rates 